\title{
XLIII. On restricted lines and planes of closest fit to systems of points in any number of dimensions
}

\section{E.C. Snow M.A.}

To cite this article: E.C. Snow M.A. (1911) XLIII. On restricted lines and planes of closest fit to systems of points in any number of dimensions, Philosophical Magazine Series 6, 21:123, 367-386, DOI: $10.1080 / 14786440308637042$

To link to this article: http://dx.doi.org/10.1080/14786440308637042

曲 Published online: 21 Apr 2009.

Submit your article to this journal $₫$

Џ Article views: 3

Q View related articles $\square$ 


\section{$\left[\begin{array}{ll}367 & ]\end{array}\right.$}

XLIII. On Restricted Lines and Planes of Closest Fit to Systems of Points in any Number of Dimensions. By E. (!. Snow, M.A.*

\section{Statement of the Problem.}

1. T WHE theory of the lines and planes of closest fit to systems of points when no restriction is placed upon those lines and planes has been developed by Prof. Pearson in various papers $t$ and is of frequent application. The connexion of these lines and planes with the formulæ of the theory of multiple correlation is indicated in those papers. If the criterion of "closest fit" is that the sum of the squares of the deviations from the line or plane measured in the direction of the "dependent" variable is to be a minimum, the equation of the line or plane is identical with the corresponding multiple correlation formula. It the sum of the squares of the deviations measured at right angles to the line or plane is a minimum (and this, from the purely geometrical point of view, is the more satisfactory criterion), the result is not of such a simple form, but the determinant from which the mean equare residual is obtained is similar to the multiple correlation determinant.

While working on certain vital statistics, it was desired to obtain a formula connecting the "dependent" variable with the "independent" variables when the values of all the variables were known at the beginning and end of a certain range, and the correlation between "dependent" and "independent" variables at all intermediate points was a maximum. Thus, if $x_{0}$ denote the "dependent" variable, and $x_{1}, x_{2}, \ldots x_{n}$ the "independent" ones, we require to make the correlation of $x_{0}$ with $x_{1}, x_{2}, \ldots x_{n}$ a maximum, with the condition that when $x_{1}, x_{2}, \ldots x_{n}$ take up the values $p_{11}, p_{21}, \ldots p_{n 1}, p_{12}, p_{22} \ldots p_{n 2}$ respectively, $x_{0}$ is to take the values $p_{01}$ and $p_{02}$.

A similar problem occurs in certain branches of Physics, especially in connexion with solutions and alloys. A property- $e$. g., the freezing-point-of a pure substance may be definitely known, and it is required to investigate the behaviour of that property as certain amounts of some other substance or substances are added. Fixed conditions will be imposed upon the law which is to be investigated by the known properties of the pure substance. The law, then,

* Communicated by Prof. Karl Pearson, F.R.S.

$\uparrow$ See Phil. Mag. Nov. 1901, pp. 559 et seqq.; Phil. Trans, vol. clxxxvii. A, pp. 301 et seqq. 
has to give the best fit to the observations made of the property as definite quantities of the other substances are added. Two examples of such cases are given from the figures of certain alloys ( $\$ 7$ below).

The idea is capable of generalization, and the theory for the general case will be investigated. Looked at from the point of view of correlation, we shall require to assume a linear law connecting $x_{0}$ with $x_{1}, x_{2}, \ldots x_{n}$, and shall make the sum of the squares of the deviations of the actual observations from this linear law measured in the direction of $x_{0}$ a minimum, making use of the exact conditions which are imposed on the law. This will be first investigated. But a better geometrical fit to the observations will be obtained by measuring the deviations perpendicular to the "plane" given by the linear law, and this will be worked out subsequently.

\section{Analytical Investigation.}

First Method.

2. Let there be $n$ "independent" variables and $(k+1)$ conditions connecting them with the "dependent" variable. $(k+1)$ is necessarily less than $n$. Measuring from one of these fixed conditions, we can assume our law is

$$
x_{0}=a_{1} x_{1}+a_{2} x_{2}+\ldots+a_{n} x_{n}, . . . .
$$

with the $k$ conditions

$$
\left.\begin{array}{c}
p_{01}=a_{1} p_{11}+a_{2} p_{21}+\ldots+a_{n} p_{n 1} \\
p_{02}=a_{1} p_{12}+a_{2} p_{22}+\ldots+a_{n} p_{n 2} \\
\cdot \cdot \cdot \cdot \cdot \cdot \cdot \cdot \cdot+a_{n} p_{n k}
\end{array}\right\} . .
$$

Then we want to make

$$
\mathrm{V}=\mathrm{S}\left(x_{0}-a_{1} x_{1}-a_{2^{2} x_{2}}-\ldots-a_{n} x_{n}\right)^{2}
$$

the sum of the square of the deviations in the direction of $x_{0}$, a minimam, subject to the above conditions.

Hence we must have

$$
\begin{array}{r}
0=\mathbb{S}\left(x_{0}-a_{1} x_{1}-a_{2} x_{2}-\ldots .-a_{n} x_{n}\right)\left(x_{1} \cdot d a_{1}+x_{2} \cdot d a_{2}\right. \\
\left.+\ldots+x_{n} \cdot d a_{n}\right),
\end{array}
$$

with the conditions

$$
0=p_{1 s} \cdot d u_{1}+p_{2 s} \cdot d \alpha_{2}+\ldots+p_{n s} \cdot d \alpha^{\prime} . \quad\left(s=1,2, \ldots k_{.}\right)(4)
$$


Planes of Closest Fit to Systems of Points.

Multiply equations (4) by $\lambda_{s}(s=1,2, \ldots k)$, and add to (3). Then, by the ordinary theory of maximum and minimum values, we know that the coefficient of $d a^{t}$ $(t=1,2, \ldots n)$ in the equation so obtained must vanish. This gives the $n$ equations

$$
\begin{aligned}
\lambda_{1} p_{t 1}+ & \lambda_{2} p_{t 2}+\ldots+\lambda_{k} p_{t k} \\
& =\mathrm{S} x_{t}\left(x_{0}-a_{1} x_{1}-a_{2} x_{2}-\ldots-a_{n} x_{n}\right) \\
& =\mathrm{R}_{0 t}-a_{1} \mathrm{R}_{1 t}-a_{2} \mathrm{R}_{2 t}-\ldots-a_{n} \mathrm{R}_{n t}, . .
\end{aligned}
$$

if $\mathrm{R}_{u v}=\mathrm{S}\left(x_{u} x_{v}\right)=\mathrm{R}_{v u}$ for all positive integral values of $u$ and $r$.

(5) gives $n$ equations connecting the $(n+k)$ unknowns, $a_{1}, a_{2}, \ldots a_{n}, \lambda_{1}, \lambda_{2}, \ldots \lambda_{k}$. (2) gives $k$ other equations between the $a$ 's, in which, however, the $\lambda$ 's are absent. Thus we have the following set of equations from which to determine the $a$ 's and the $\lambda^{\prime} \mathrm{s}:-$

$$
\begin{aligned}
& a_{1} \mathrm{R}_{1 t}+a_{2} \mathrm{R}_{2 t}+\ldots+a_{n} \mathrm{R}_{n t}+\lambda_{1} p_{t 1}+\lambda_{2} p_{t 2}+\ldots+\lambda_{k} p_{t k}=\mathrm{R}_{0 t} \text {. } \\
& (t=1,2, \ldots . n \text {.) } \\
& a_{1} p_{1 s}+a_{2} p_{2 s}+\ldots+a_{n} p_{n s} \\
& (s=1,2, \ldots k)=p_{0 s} \text {. }
\end{aligned}
$$

Let $\Delta$ denote

$$
\begin{array}{cccccccc}
\mathrm{R}_{00} & \mathrm{R}_{10} & \ldots & \mathrm{R}_{n 0} & p_{01} & p_{02} & \ldots & p_{0 k} \\
\mathrm{R}_{01} & \mathrm{R}_{11} & \ldots & \mathrm{R}_{n 1} & p_{11} & p_{12} & \ldots & p_{1 k} \\
\vdots & \vdots & & \vdots & \vdots & \vdots & & \vdots \\
\mathrm{R}_{0 n} & \mathrm{R}_{1 n} & \ldots & \mathrm{R}_{n n} & p_{n 1} & p_{n 2} & \ldots & p_{n k} \\
p_{01} & p_{11} & \ldots & p_{n 1} & 0 & 0 & \ldots & 0 \\
p_{02} & p_{12} & \ldots & p_{n 2} & 0 & 0 & \ldots & 0 \\
\vdots & \vdots & & \vdots & \vdots & \vdots & & \vdots \\
p_{0 k} & p_{1 k} & \ldots & p_{n k} & 0 & 0 & \ldots & 0
\end{array}
$$

a determinant of the order $(n+k+1)$.

Then the solutions of equations (6) are :

and

$$
a_{t}=-\frac{\Delta_{t 0}}{\Delta_{00}} \quad(t=1,2, \ldots n) \quad . \quad .
$$

$$
\lambda_{s}=-\frac{\Delta_{(u+s), 0}}{\Delta_{(n, j)}}(s=1,2, \ldots k) \quad . \quad .
$$


$\Delta_{u v}$ being the first minor of the constituent of the $(u+1)$ th column and $(v+1)$ th row, and being positive or regative according as $(u+v)$ is even or odd.

Thus the coefficients in the required formula can be found at once by the evaluation of a number of determinants of order $(n+k)^{*}$.

\section{Particular Cases.}

3. The simplification of the above results in a few particular cases will be useful.

(A) $k=0$. - In this case we have a plane passing through a single fixed point and closest fitting to a system of points. Here all the $\lambda$ 's disappear, and the equation of the plane becomes

where

$$
x_{0}=a_{1} x_{1}+a_{2} x_{2}+\ldots+a_{n} x_{n}
$$

$\Delta$ buing

$$
a_{t}=-\frac{\Delta_{0 t}}{\Delta_{00}}
$$

$$
\left|\begin{array}{cccccc}
\mathrm{R}_{00} & \mathrm{R}_{10} & \ldots & \mathrm{R}_{s 0} & \ldots & \mathrm{R}_{n 0} \\
\mathrm{R}_{01} & \mathrm{R}_{11} & \ldots & \mathrm{R}_{s 1} & \ldots & \mathrm{R}_{n 1} \\
\mathrm{R}_{02} & & & & \vdots \\
\vdots & & & & \vdots \\
\mathrm{R}_{0 n} & \ldots & \ldots & \ldots & \vdots \\
\mathrm{R}_{n n}
\end{array}\right|
$$

$\mathrm{R}_{u v}$ being equal to $\mathbf{R}_{v u}$, and is the sum of the products $x_{u} . x_{v}$ taken throughout the system of points.

If $\hat{x}_{s}$ and $\sigma_{s}$ be the mean and standard deviation of $x_{s}$, and $r_{u v}$ the correlation between the coordinates $x_{u}$ and $x_{v}$, we have

$$
\begin{aligned}
& \mathrm{R}_{u v}=\mathrm{S}\left(x_{u} x_{v}\right)=\mathrm{N} \sigma_{u} \sigma_{v} r_{u v}+\mathrm{N} \bar{x}_{u} \bar{x}_{v} \text { if } u \neq v, \\
& \text { and } \quad \mathrm{R}_{u v}=\mathrm{N} \sigma_{u}{ }^{2}+\mathrm{N} \bar{x}_{u}{ }^{2} \text { if } u=v .
\end{aligned}
$$

The analogy between $\Delta$ and the determinant used in the

* It is not difficult to show that, by first finding the $a$ 's in terms of the $\lambda$ 's from the first $n$ of equations (6), and substituting these values in the last $k$ of the same set-thus giving $k$ equations for the $\lambda$ 's, - the $a^{\prime}$ s can be found in a form involving only deteiminants of order $n$, though the number of determinants it is necessary to evaluate is increased. If $k$ is large, this increase is considerable. The general result in this form will not be given, but it is exemplified in a particular case below $(\$ 3)$. 
theory of multiple correlation is now clear, for $a_{t}$ can be written $-\frac{\Delta_{t 0}^{\prime}}{\Delta_{00}^{\prime}}$, where

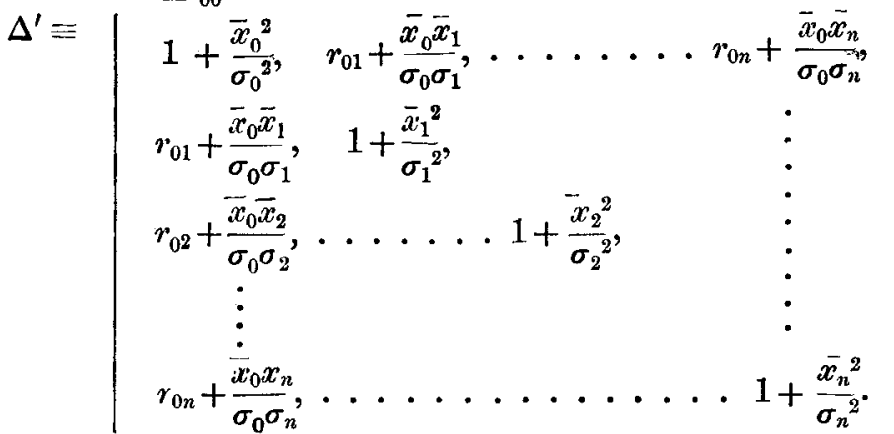

Thus this determinant can be derived from the multiple correlation determinant by increasing $r_{s t}$ in the latter by $V_{s} . V_{t}$, and by increasing the constituents of the leading term by the corresponding $\mathrm{V}_{s}^{2}$, where $\mathrm{V}_{s}$ and $\mathrm{V}_{t}$ are the coefficients of variation of the coordinates $x_{s}$ and $x_{t}$. If the fixed point is at the mean of the system of given points, $\Delta^{\prime}$ becomes at once the ordinary multiple correlation determinant.

Putting $n=1$, we derive the two-dimensional case of a line passing through the origin and giving closest fit (measuring in the direction of $y$ ) to a system of points. The equation of the line is easily seen to be

$$
y=\frac{\mathrm{R}_{10}}{\mathrm{R}_{11}} x=\frac{\mathrm{S}(x \cdot y)}{\mathrm{S}\left(x^{2}\right)} x . . . . .
$$

Putting $n=2$, we reach the three-dimensional case of a plane passing through the origin and giving closest fit (measured in the direction of $z$ ) to a system of points. Its equation is

$$
\begin{aligned}
\mathrm{Z} & =\frac{\mathrm{R}_{12} \mathrm{R}_{02}-\mathrm{R}_{01} \mathrm{R}_{22}}{\mathrm{R}_{11} \mathrm{R}_{22}-\mathrm{R}_{12}{ }^{2}} \cdot x+\frac{\mathrm{R}_{12} \mathrm{R}_{01}-\mathrm{R}_{02} \mathrm{R}_{11}}{\mathrm{R}_{11} \mathrm{R}_{22}-\mathrm{R}_{12}{ }^{2}} y \\
& =\frac{\mathrm{S}(x y) \cdot \mathrm{S}(y z)-\mathrm{S}(x z) \cdot \mathrm{S}\left(y^{2}\right)}{\mathrm{S}\left(x^{2}\right) \cdot \mathrm{S}\left(y^{2}\right)-\{\mathrm{S}(x y)\}^{2}} x+\frac{\mathrm{S}(x y) \cdot \mathrm{S}(x z)-\mathrm{S}(y z) \cdot \mathrm{S}\left(x^{2}\right)}{\mathrm{S}\left(x^{2}\right) \cdot \mathrm{S}\left(y^{2}\right)-\{\mathrm{S}(x y)\}^{2}} y .
\end{aligned}
$$

For values of $n>2$ it is more convenient to derive the coefficients direct from the determinant, and there is no need to write them in full.

(B) $k=1$.-Here we have the case of a plane--in $n$ dimensions-passing through two fixed points and fitting most 
closely a system of other points. We have in this case

$$
\Delta=\left|\begin{array}{ccccc}
\mathrm{R}_{00} & \mathrm{R}_{10} & \ldots & \mathrm{R}_{n 0} & p_{0} \\
\mathrm{R}_{01} & \mathrm{R}_{11} & \ldots & \mathrm{R}_{n 1} & p_{1} \\
\vdots & \vdots & & \vdots & \vdots \\
\mathrm{R}_{0 n} & \mathrm{R}_{1 n} & \ldots & \mathrm{R}_{n n} & p_{n} \\
p_{0} & p_{1} & \ldots & p_{n} & 0
\end{array}\right| \text {, }
$$

$p_{0}, p_{1}, \ldots p_{n}$ being the coordinates of one fixed point relative to the other, which is taken as origin.

The coefficients in the required equation can be found from the above in the form of determinants of order $(n+1)$.

But in this case the first $n$ equations of (6) become

$$
a_{1} \mathrm{R}_{1 t}+\ldots+a_{n} \mathrm{R}_{n t}=\mathrm{R}_{0 t}-\lambda p_{t} \quad(t=1,2, \ldots n) .
$$

Solving these for $a_{1}, a_{2}, \ldots a_{n}$ in terms of $\lambda$, we have

where

$$
a_{t}=-\frac{\delta_{0 t}}{\delta_{00}}
$$

where

$$
\begin{aligned}
\delta & \equiv\left|\begin{array}{cccc}
\mathrm{R}_{00} & \mathrm{R}_{01} & \ldots & \mathrm{R}_{0 n} \\
\mathrm{R}_{01}-\lambda p_{1} & \mathrm{R}_{11} & \ldots & \mathrm{R}_{1 n} \\
\vdots & \vdots & & \vdots \\
\mathrm{R}_{0 n}-\lambda p_{n} & \mathrm{R}_{1 n} & \ldots & \mathrm{R}_{n n}
\end{array}\right| \\
& =\delta^{\prime}-\lambda \delta^{\prime \prime},
\end{aligned}
$$

and

$$
\delta^{\prime} \equiv\left|\begin{array}{cccc}
\mathrm{R}_{00} & \mathrm{R}_{01} & \ldots & \mathrm{R}_{0 n} \\
\mathrm{R}_{01} & \mathrm{R}_{11} & \ldots & \mathrm{R}_{1 n} \\
\vdots & \vdots & & \vdots \\
\mathrm{R}_{0 n} & \mathrm{R}_{1 n} & \ldots & \mathrm{R}_{n n}
\end{array}\right|
$$

$$
\delta^{\prime \prime} \equiv\left|\begin{array}{cccc}
1 & \mathrm{R}_{01} & \ldots & \mathrm{R}_{0 n} \\
p_{1} & \mathrm{R}_{11} & \ldots & \mathrm{R}_{1 n} \\
p_{2} & \vdots & & \vdots \\
\vdots & \vdots & & \vdots \\
p_{n} & \mathrm{R}_{1 n} & \ldots & \mathrm{R}_{n n}
\end{array}\right|
$$

so that

$$
a_{t}=-\frac{\delta_{0 t}^{\prime}-\lambda \delta_{0 t}^{\prime \prime}}{\delta_{t(v)}} \quad(t=1,2, \ldots n) \text {. }
$$


When these values are substituted in the equation giving the fixed condition, viz.

$$
p_{0}=a_{1} p_{1}+\ldots+\alpha_{t} p_{t}+\ldots+a_{n} p_{n}
$$

$\lambda$ can be found, and from this the $a$ 's can be completely determined. This method will be used in an example below.

No other particular cases of this method need be worked out in detail. We see that it is always possible to obtain a plane in $n$-dimensional space to pass through any number (less than $n$ ) of fixed points and to be such that the sum of the squares of the deviations of any number of other points from the plane measured in a fixed direction is a minimum.

Second Method.

4. We have now to investigate the equation of the corresponding plane when the criterion for "closest fit" is that the sum of the squares of the deviations from the plane measured at right angles to the plane is the least possible. From a purely geometrical point of view this will give a closer plane to the system of points, but it will not give the regression of one variable on the others.

Let $l_{1} \ldots l_{n}$ be the generalized direction-cosines of the plane, and take one of the points through which the plane has to pass as origin.

Then the equation of the plane is

$$
l_{1} x_{1}+\ldots .+l_{n} x_{n}=0, \text {. . . . . }
$$

the total number of variables being taken as $n$ for convenience of notation. There will also be the conditions

and

$$
l_{1}^{2}+\ldots .+l_{n}^{2}=1 \text {. . . . }
$$

$(k+1)$ being the total number of fixed conditions.

The criterion for closest fit is

$$
\mathrm{V}=\mathrm{S}\left(l_{1} x_{1}+\ldots+l_{n} x_{n}\right)^{2}
$$

to be minimum, subject to the above conditions.

Hence

$$
0=\mathrm{S}\left(l_{1} x_{1}+\ldots+l_{n} x_{n}\right)\left(x_{1} d l_{1}+\ldots+x_{n} d l_{n}\right),
$$

together with

$$
\begin{gathered}
l_{1} d l_{1}+\ldots+l_{n} d l_{n}=0 \\
p_{11} d l_{1}+\ldots+p_{n 1} d l_{n}=0, \\
p_{1 k} d \vec{l}_{1}+\ldots+\dot{p}_{n k} d l_{n}=0 .
\end{gathered}
$$


The conditions for a minimum give

$$
\left.\begin{array}{c}
\mathrm{X}_{1}+\mu l_{1}+\mu_{1} p_{11}+\mu_{2} p_{12}+\ldots+\mu_{k} p_{1 k}=0, \\
\ldots \ldots+\cdots \\
\mathrm{X}_{n}+\mu l_{n}+\mu_{1} p_{n 1}+\mu_{2} p_{n 2}+\ldots+\mu_{k} p_{n k}=0,
\end{array}\right\} .
$$

where $\mu, \mu_{1}, \ldots \mu_{k}$ are undetermined constants, and

$$
\begin{aligned}
\mathrm{X}_{s} & =\mathrm{S}\left(l_{1} x_{1}+\ldots+l_{n} x_{n}\right) x_{s} \\
& =l_{1} \mathrm{R}_{1 s}+\ldots+l_{n} \mathrm{R}_{n s},
\end{aligned}
$$

where, as before,

$$
\mathrm{R}_{u v}=\mathrm{S}\left(x_{u}, x_{v}\right)=\mathrm{R}_{v, u} .
$$

Multiply equations $(12)$ by $l_{1}, l_{2}, \ldots l_{n}$ respectively, and add. Remembering the conditions (10) and (11), we at once obtain

$$
\mu+l_{1} \mathrm{X}_{1}+l_{2} \mathrm{X}_{2}+\ldots+l_{n} \mathrm{X}_{n}=0,
$$

where $l_{1} \ldots l_{n}$ have the values which make $V$ a minimum. But then $l_{1} \mathrm{X}_{1}+l_{q} \mathrm{X}_{2}+\ldots+l_{n} \mathrm{X}_{n}$ becomes $\mathrm{V}_{m}^{*}$, the minimum value of $V$.

It follows that

$$
\mu=-\mathrm{V}_{m} \text {. }
$$

Substituting this value of $\mu$ in (12), we shall have with (11) $(n+k)$ equations between $l_{1} \ldots l_{n}, \mu_{1} \ldots \mu_{k}$ and $V_{m}$. Hence we can eliminate the $l$ 's and the $\mu$ 's and obtain an equation to determine $V_{m}$.

Since

$$
\mathrm{X}_{s}=l_{1} \mathrm{R}_{1 s}+\ldots+l_{n} \mathrm{R}_{n s},
$$

this eliminant can be written in the determinantal form

$$
\mathrm{D} \equiv\left|\begin{array}{cccccccc}
\mathrm{R}_{11}-\mathrm{V}_{m} & \mathrm{R}_{21} & \ldots & \mathrm{R}_{n 1} & p_{11} & p_{12} & \ldots & p_{1 k} \\
\mathrm{R}_{12} & \mathrm{R}_{22}-\mathrm{V}_{m} & \ldots & \mathrm{R}_{n 2} & p_{21} & p_{22} & \ldots & p_{2 k} \\
\vdots & \vdots & & \vdots & \vdots & \vdots & & \vdots \\
\mathrm{R}_{1 n} & \mathrm{R}_{2 n} & \ldots & \mathrm{R}_{n n}-\mathrm{V}_{m} & p_{n 1} & p_{n 2} & \ldots & p_{n k} \\
p_{11} & p_{21} & \ldots & p_{n 1} & 0 & 0 & \ldots & 0 \\
p_{12} & p_{22} & \ldots & p_{n 2} & 0 & 0 & \ldots & 0 \\
\vdots & \vdots & & \vdots & \vdots & \vdots & & \vdots \\
p_{1 k} & p_{2 k} & \ldots & p_{n k} & 0 & 0 & \ldots & 0
\end{array}\right|=0 .
$$

This is an equation of the $(n-k)$ th degree in $V_{m}$. Its roots are necessarily positive (being the sum of a number of 
squares of real quantities), and the smallest of these must be taken. When this value of $\mathrm{V}_{m}$ is substituted in (11) and (12), $(n+k)$ homogeneous equations in $l_{1} l_{2} \ldots l_{n}, \mu_{1} \mu_{2} \ldots \mu_{k}$ are obtained, $(n+k-1)$ of which, together with (10), suffice to determine all the $l$ 's and the $\mu$ 's. As before, this can be done in determinant form, the order of the determinants involved being $(n+k-1)$.

\section{Particular Cases.}

5. Useful particular cases of the general formula are obtained by taking $k=0$ and $k=1$.

$\left(\mathrm{A}^{\prime}\right)$ If $k=0$, we derive the case analogous to (A) above. The equation to determine $V_{m}$ takes the well-known form

$$
\left|\begin{array}{cccc}
\mathrm{R}_{11}-\mathrm{V}_{m} & \mathrm{R}_{21} & \ldots & \mathrm{R}_{n 1} \\
\mathrm{R}_{12} & \mathrm{R}_{22}-\mathrm{V}_{m} & \ldots & \mathrm{R}_{n 2} \\
\vdots & \vdots & & \vdots \\
\mathrm{R}_{1 n} & \mathrm{R}_{2 n} & \ldots & \mathrm{R}_{n n}-\mathrm{V}_{m}
\end{array}\right|=0
$$

Putting $n=2$, we have the two-dimensional case, and $\mathrm{V}_{m}$ is the least root of the quadratic

so that

$$
\left|\begin{array}{cc}
\mathrm{R}_{11}-\mathrm{V}_{m} & \mathrm{R}_{21} \\
\mathrm{R}_{12} & \mathrm{R}_{22}-\mathrm{V}_{m}
\end{array}\right|=0 ;
$$

and

$$
\begin{array}{r}
2 \mathrm{~V}_{m}==\left(\mathrm{R}_{11}+\mathrm{R}_{22}\right)-\left\{\left(\mathrm{R}_{11}-\mathrm{R}_{22}\right)^{2}+4 \mathrm{R}_{12}{ }^{2}\right\}^{1 / 2}, \\
\text { since } \mathrm{R}_{12}=\mathrm{R}_{21},
\end{array}
$$

$$
\begin{aligned}
& 2\left(\mathrm{R}_{11}-\mathrm{V}_{m}\right)=\left(\mathrm{R}_{11}-\mathrm{R}_{22}\right)+\left\{\left(\mathrm{R}_{11}-\mathrm{R}_{22}\right)^{2}+4 \mathrm{R}_{12}{ }^{2}\right\}^{1 / 2} . \\
& \text { Put } \\
& \frac{\mathrm{R}_{11}-\mathrm{R}_{22}}{\cos \theta}=\frac{2 \mathrm{R}_{12}}{\sin \theta}=\rho .
\end{aligned}
$$

Then

$$
2\left(\mathrm{R}_{11}-\mathrm{V}_{m}\right)=\rho(1+\cos \theta)=2 \rho \cos ^{2} \frac{\theta}{2}
$$

and

$$
2 \mathrm{R}_{12}=2 \rho \sin \frac{\theta}{2} \cos \frac{\theta}{2} \text {. }
$$

The first of equations (12) now gives

$$
l_{1} \cos \frac{\theta}{2}+l_{2} \sin \frac{\theta}{2}=0
$$

since the $p$ 's vanish when $k=0$. 
The equition of the line, therefore, is

$$
x \sin \frac{\theta}{2}+y \cos \frac{\theta}{2}=0 \text {, }
$$

where

$$
\begin{aligned}
\tan \theta & =\frac{2 \mathrm{R}_{12}}{\mathrm{R}_{11}-\mathrm{R}_{22}} \\
& =\frac{2 \mathrm{~S}(u y)}{\mathrm{S}\left(x^{2}\right)-\mathrm{S}\left(y^{2}\right)},
\end{aligned}
$$

and the notation is altered to agree with the usual form. This value of $\tan \theta$ gives rise to two values of $\frac{\theta}{2}$, each less than $180^{\circ}$. In a particular numerical example, however, it is not difficult to pick out the value required; while it can be verified that the other value corresponds to the "worstfitting "line.

In cases of $n>2$ it is better to substitute the values of the $R$ 's direct in the determinant above, and to find $V_{m}$ by the usual methods of approximating to the roots of an equation.

$\left(B^{\prime}\right)$ If $k=1$, the equation to determine $V_{m}$ is

$$
d=\left|\begin{array}{ccccc}
\mathrm{R}_{11}-\mathrm{V}_{m} & \mathrm{R}_{21} & \ldots & \mathrm{R}_{n 1}^{\prime}, & p_{1} \\
\mathrm{R}_{12} & \left(\mathrm{R}_{22}-\mathrm{V}_{m}\right) & \ldots & \mathrm{R}_{n \dot{y}}, & p_{2} \\
\vdots & \vdots & & \vdots & \vdots \\
\mathrm{R}_{1 n} & \mathrm{R}_{2 n} & \ldots & \left(\mathrm{R}_{n n}-\dot{\mathrm{V}}_{n}\right), & p_{n} \\
p_{1} & p_{2} & \ldots & p_{n}, & 0
\end{array}\right|=0
$$

the origin and the point $\left(p_{1} p_{2} \ldots p_{n}\right)$ being the fixed points.

In this case, equations (12) take the form

$$
\begin{aligned}
l_{1}\left(\mathrm{R}_{11}-\mathrm{V}_{m}\right)+ & l_{2} \mathrm{R}_{21}+\ldots+l_{n} \mathrm{R}_{n 1} \\
\cdots \cdots+\cdots & =-\lambda p_{1}, \\
l_{1} \mathrm{R}_{1 n}+l_{2} \mathrm{R}_{2 n}+\ldots+l_{n}\left(\mathrm{R}_{n n}-\mathrm{V}_{m}\right) & =-\lambda p_{n} .
\end{aligned}
$$

Hence $l_{t}$ is proportional to $d_{t}$, the first minor of the constituent in the $t \mathrm{tb}$ column and bottom row of $d$, and $\mathrm{V}_{m}$ is given the value which is the least root of $d=0$. Using (10), the actual values of the $l$ 's can be found.

6. It will be seen from the foregoing analysis that the work involved in determining the "closest fitting " plane by 
the second criterion is much greater, at any rate in all cases of $n>2$, than that necessitated by the first criterion. In the second method, if $(n-k)$ is three or more, the smallest root of an equation of the third or higher degree has to be approximated to. This in itself is no light task, and is not necessary in the first method. The methods do not necessarily lead to results at all alike (see Example 3, below), and only the terms of the particular question in hand can decide which method is to be used. The second gives the best geometrical fit, considered in a direction perpendicular to the plane. The first gives the "regression" plane-i.e., the most probable value of one variable in terms of the others. This is the most frequently needed in practical cases, as is exemplified in Examples 1 and 2.

\section{Illustrations.}

I. The second column in Table I.* gives the temperatures (Centigrade) at solidification of a series of alloys of iron and

\section{TABLE I.}

\begin{tabular}{|c|c|c|c|}
\hline $\begin{array}{c}x . \\
\text { Percentage } \\
\text { of Carbon } \\
\text { present. }\end{array}$ & $\begin{array}{l}y . \\
\text { Temperature } \\
\text { at end of } \\
\text { aolidificution. }\end{array}$ & $\begin{array}{l}\text { Temperature by } \\
\text { lst method to } \\
\text { neurest degree. }\end{array}$ & $\begin{array}{l}\text { Temperature by } \\
\text { 2nd mothod to } \\
\text { nearest degree. }\end{array}$ \\
\hline $\begin{array}{r}\cdot 02 \\
\cdot 12 \\
\cdot 16 \\
\cdot 17 \\
.24 \\
.38 \\
.53 \\
.61 \\
\cdot 80 \\
\cdot 81 \\
1 \cdot 31 \\
1 \cdot 51 \\
1 \cdot 85 \\
2 \cdot 12 \\
2 \cdot 21\end{array}$ & $\begin{array}{l}1470 \\
1470 \\
1465 \\
1450 \\
1448 \\
1416 \\
1404 \\
1394 \\
1351 \\
1351 \\
1286 \\
1244 \\
1179 \\
1110 \\
1107\end{array}$ & $\begin{array}{l}1501 \\
1483 \\
1476 \\
1474 \\
1461 \\
1436 \\
1409 \\
1395 \\
1360 \\
1358 \\
1268 \\
1231 \\
1171 \\
1122 \\
1105\end{array}$ & $\begin{array}{l}1501 \\
1483 \\
1476 \\
1474 \\
1461 \\
1436 \\
1409 \\
1394 \\
1359 \\
1357 \\
1267 \\
1230 \\
1169 \\
1120 \\
1103\end{array}$ \\
\hline
\end{tabular}

carbon. The percentage of carbon in the various alloys is given in the first column. The solidifying temperature of pure iron is $1505^{\circ} \mathrm{C}$. Any curve, therefore, which attempts

* The table is taken from a paper on "The Range of Solidification and the Critical Ranges of Iron-Carbon Alloys," by H. C. H. Carpenter, M.A., and B. F. E. Keeling, B.A., in the Journal of the Iron and Steel Institute, No. 1, for 1904 .

Phil. Mag. S. 6. Vol. 21. No. 123. Marrh 1911. 2 C 
to express a relationship between the percentage of carbon present and the solidifying point of the alloy should pass through the fixed point $\left(0 \%, 1505^{\circ} \mathrm{C}\right.$.). $\mathrm{Up}$ to $2 \%$ of carbon a straight line seems to be the most likely fit. The two methods will therefore be applied to get a line to pass through the point $\left(0 \%, 1505^{\circ} \mathrm{C}\right.$.) and to fit the series of observations. It will be seen from the figure that up to a percentage of carbon of $0.5 \%$ the results are irregular, but from that point up to $2 \%$ the irregularities are small and within the limits of experimental error.

Measuring $x$ positively from zero and $y$ negatively from 1505 , we find

$$
\begin{aligned}
& S\left(x^{2}\right)=19 \cdot 0176, \\
& \mathbf{S}\left(x_{y}\right)=3438 \cdot 41, \\
& \mathbf{S}\left(y^{2}\right)=624986 .
\end{aligned}
$$

By, the first method the equation of the line (neasured from $0 \%$ and $1505^{\mathrm{c}}$ (.) is

$$
y=\frac{\mathrm{S}(x \cdot y)}{\mathrm{S}\left(x^{2}\right)} \cdot x \quad \text { or } \quad y=180 \cdot 801 x .
$$

The relationship between the temperature of solidification and percentage of carbon present is therefore

$$
\mathrm{T}=1505-180.801 x \text {. }
$$

If we apply the second method, we find

$$
\tan \theta=-.0110035
$$

whence

$$
\frac{\theta}{2}=89^{\circ} 41^{\prime} 5^{\prime \prime} \cdot 23
$$

and

$$
\tan \frac{\theta}{2}=181 \cdot 80843
$$

which gives the relationship

$$
\mathrm{T}=1505-181 \cdot 808 x .
$$

The actual temperatures obtained from the two formula are given in columns 3 and 4 of Table I. We see that, to the nearest degree, there is no difference in the results up to $0.5 \%$ of carbon, and the difference beyond that point is small. The two lines cannot be distinguished on a diagram of the size shown. The line $\mathrm{OP}$ in the diagram represents, 
therefore, the best fit to the system of points of a line through $\mathrm{O}$ by both methods.

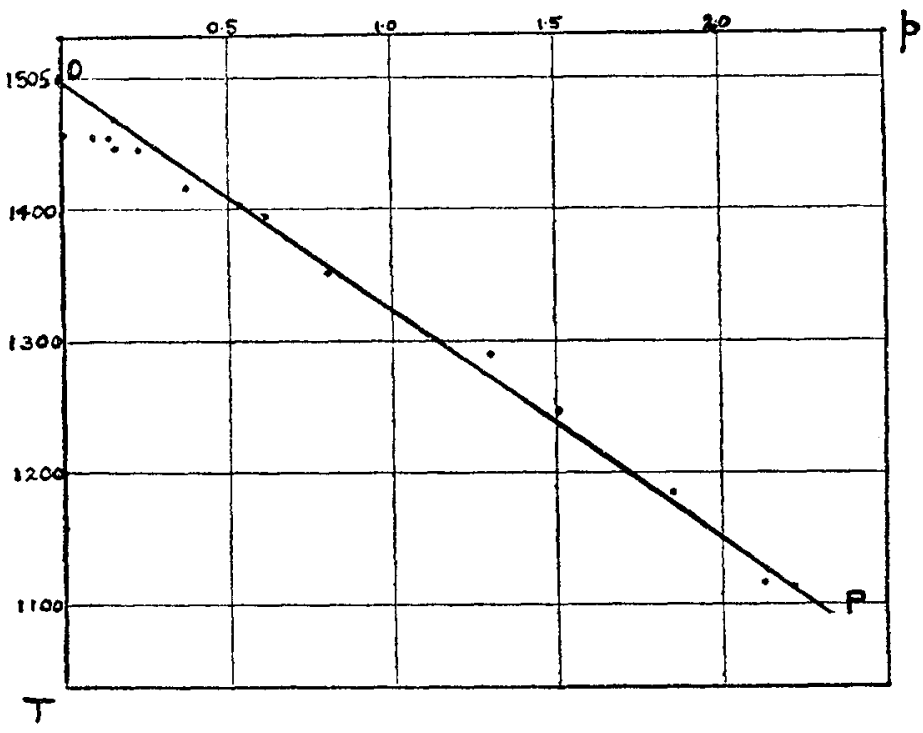

II.* In this case we will take the figures of an alloy of three metals-copper, aluminium, and manganese. The percentage of mangazese present varies from 0 to 10.24 , and of aluminiun from 0 to $7 \cdot 40$. It is not possible to tell from the mere figures if the distribution is approximately coplanar or not, but the material seemed good enough to work upon. The freezing-point of pure copper is $1084^{\circ} \mathrm{C}$.; in this case, therefore, we require to find a relationsbip of the form

$$
\mathrm{T}-1084=p \cdot x+q \cdot y,
$$

where $\mathrm{T}$ is the temperature at solidification of an alloy containing $x \%$ of aluminium and $y \%$ of manganese. Taking our origin at zero percentages of aluminium and manganese. and $1084^{\circ} \mathrm{C}$., we require to obtain a plane through the origin and fitting most closely the observations in the second, third, and fourth columns of Table II.

* The figures for this example were taken from Table 43 (p. 229) of the "Ninth Report to the Alloys Research Committee" to the Inst. of Mechanical Encrineers, by Jr. W. Rosenhain and Mr. F. C. A. H. Lantsbery. 
TABLE II.

\begin{tabular}{|c|c|c|c|c|c|c|c|}
\hline $\begin{array}{c}\text { Actual } \\
\text { Freezing- } \\
\text { Point. } \\
{ }^{\circ} \mathrm{O} .\end{array}$ & $\begin{array}{c}\text { Deviation } \\
\text { of Temp. } \\
\text { from } \\
1084^{\circ} \mathrm{O} .\end{array}$ & $\begin{array}{c}\text { Percentage } \\
\text { of } \\
\text { Alnminium } \\
\text { present. }\end{array}$ & $\begin{array}{c}\text { Percentage } \\
\text { of } \\
\text { Manganese } \\
\text { present. }\end{array}$ & $\begin{array}{c}\text { Temp. by } \\
\text { Formula } \\
(y) .\end{array}$ & $\begin{array}{c}\text { Deriation } \\
\text { from } \\
\text { Aetual } \\
\text { Temp. }\end{array}$ & $\begin{array}{l}\text { Temp. by } \\
\text { Formula } \\
(\epsilon) .\end{array}$ & $\begin{array}{c}\text { Deviation } \\
\text { from } \\
\text { Actual } \\
\text { Temp. }\end{array}$ \\
\hline 1077 & 7 & $1 \cdot 19$ & $1 \cdot 14$ & $1067 \cdot 8$ & $-9 \cdot 2$ & $1067 \cdot 7$ & $-9 \cdot 3$ \\
\hline 1045 & 39 & $1 \cdot 37$ & $2 \cdot 75$ & $1053 \cdot 2$ & $+8 \cdot 2$ & $1052 \cdot 9$ & $+7 \cdot 9$ \\
\hline 1051 & 33 & 1.04 & 4.92 & $10: 366$ & $-14 \cdot 4$ & $1036 \cdot 6$ & $-14 \cdot 4$ \\
\hline 1023 & 6J & $1 \cdot 42$ & $5 \cdot 38$ & 10307 & $+7 \cdot 7$ & $1030 \cdot 2$ & $+7 \cdot 2$ \\
\hline 1015 & 69 & 0.94 & $6 \cdot 48$ & 10240 & +90 & 1023.4 & $+8 \cdot 4$ \\
\hline 1007 & 77 & $1 \cdot 43$ & $7 \cdot 72$ & $1010 \cdot 8$ & +38 & $1010 \cdot 8$ & +38 \\
\hline 1011 & 73 & 0.91 & $8 \cdot 16$ & $1012 \cdot 1$ & +1.1 & $1009 \cdot 2$ & $-1 \cdot 8$ \\
\hline 985 & 98 & 1.91 & $10 * 24$ & $987 \cdot 2$ & $+2 \cdot 2$ & $985 \cdot 9$ & +0.9 \\
\hline 1075 & 9 & 269 & & $1069 \cdot 3$ & -57 & $1069 \cdot 2$ & -58 \\
\hline 1057 & 27 & $2 \cdot 36$ & $1 \cdot 95$ & $1054 \cdot 6$ & $-2 \cdot 4$ & $1054 \cdot 4$ & $-2 \cdot 6$ \\
\hline 1045 & 39 & 231 & $3 \cdot 82$ & $1039 \cdot 0$ & $-6 \cdot 0$ & $1038 \cdot 6$ & $-6 \cdot 4$ \\
\hline 1010 & 74 & $2 \cdot 27$ & $7 \cdot 80$ & $1006 \cdot 5$ & $-3 \cdot 5$ & $1004 \cdot 2$ & -5.2 \\
\hline 896 & 88 & 237 & $9 \cdot 76$ & 9884 & $-7 \cdot 6$ & 987.5 & -8.5 \\
\hline 1059 & 25 & $3 * 26$ & 0.97 & 10580 & -1.0 & $1057 \cdot 8$ & $-1 \cdot 2$ \\
\hline 1042 & 42 & $3 \cdot 71$ & $2 \cdot 97$ & 1038.6 & -34 & $103 \times 2$ & $-3 \cdot 8$ \\
\hline 1043 & 41 & 3.93 & 299 & $1037 \cdot 2$ & -5.8 & 1036.8 & $-6 \cdot 2$ \\
\hline 1024 & 60 & 329 & $4 \cdot 80$ & $1025 \cdot 4$ & +1.4 & $1023 \cdot 1$ & +0.9 \\
\hline 1022 & 62 & 3.57 & $5 \cdot 64$ & $1016 \cdot 7$ & -5.3 & 1016.1 & -5.9 \\
\hline 1015 & 69 & 308 & 6.88 & $1008 \cdot 9$ & $-6 \cdot 1$ & $1008 \cdot 2$ & $-6 \cdot 8$ \\
\hline 995 & 89 & 395 & $7 \cdot 95$ & $995 \cdot 1$ & +0.1 & $994 \cdot 3$ & $-v \cdot 7$ \\
\hline 1067 & 17 & 467 & & $1058 \cdot 5$ & $-8 \cdot 5$ & $1058 \cdot 4$ & $-8 \cdot 6$ \\
\hline 1054 & 30 & $4 \cdot 14$ & $1 \cdot 77$ & 1046.4 & $-7 \cdot 6$ & $1046 \cdot 1$ & -7.9 \\
\hline 1031 & 53 & 462 & $3 \cdot 26$ & $1031 \cdot 1$ & $+0 \cdot 1$ & $1030 \cdot 7$ & -03 \\
\hline 1036 & 48 & $4 \cdot 48$ & $3 \cdot 86$ & 1026.8 & $-9 \cdot \overline{2}$ & $1027 \cdot 4$ & $-9 \cdot 6$ \\
\hline 987 & 97 & $4 \cdot 51$ & 774 & 993.8 & $+6 . \overline{8}$ & 493.0 & +60 \\
\hline 975 & 109 & $4 \cdot 12$ & $9 \cdot 60$ & 980.2 & $+5 \cdot 2$ & $979 \cdot 2$ & $+4 \cdot 2$ \\
\hline 3050 & 34 & $5 \cdot 21$ & 0.08 & $1047 \cdot 3$ & -27 & $1047 \cdot 0$ & -30 \\
\hline 1035̃ & 49 & 566 & $2 \cdot 58$ & $1081 \cdot 2$ & $-3 \cdot 8$ & $1030 \cdot 8$ & $-4 \cdot 2$ \\
\hline 1018 & 66 & $5 \cdot 21$ & 4.86 & $1014 \cdot 4$ & $-3 \cdot 6$ & 1013.8 & $-4 \cdot 2$ \\
\hline 1001 & 83 & $5 \cdot 86$ & 6.00 & $1001 \cdot 2$ & $+0 \cdot 2$ & $1005 \cdot 5$ & $-0 \%$ \\
\hline 998 & 86 & $5 \cdot 19$ & 672 & $998 \cdot \overline{7}$ & +0.7 & 9980 & 00 \\
\hline 986 & 98 & 562 & $8 \cdot 50$ & 9827 & $-3 \cdot 3$ & $980 \cdot 4$ & -56 \\
\hline 976 & 108 & 5.99 & 9.50 & $970 \cdot 8$ & $-5 \cdot 2$ & $969 . \overline{8}$ & $-6 \cdot 2$ \\
\hline 1025 & 59 & 688 & 0.98 & $1038 \cdot 1$ & $+13 \cdot 1$ & $1087 \cdot 8$ & $+12 \cdot 8$ \\
\hline 1030 & 54 & 6.54 & 1.98 & $1031 \cdot 5$ & $+1 \cdot 5$ & $1031 \cdot 1$ & $+1 \cdot 1$ \\
\hline 1022 & 62 & 6.29 & 350 & 10200 & -20 & $1019 \cdot 5$ & $-2 \cdot 5$ \\
\hline 995 & 89 & $6 \cdot 62$ & 482 & $1007 \cdot 0$ & $+12 \cdot 0$ & $1006 \cdot 4$ & $+11 \cdot 4$ \\
\hline 978 & 106 & $6 \cdot 26$ & 802 & $981 \cdot 9$ & +39 & $981 \cdot 0$ & $+3 \cdot 0$ \\
\hline 957 & 127 & 6.91 & 9.06 & $969 \cdot 5$ & $+12 \cdot 5$ & $968 \cdot 5$ & $+11 \cdot 5$ \\
\hline 1042 & 42 & $7 \cdot 40$ & ....... & 10436 & +16 & $1043 \cdot 4$ & +14 \\
\hline
\end{tabular}

If $z$ denote deviation of the temperature from $1084^{\circ} \mathrm{C}$., we find

$$
\begin{array}{ll}
\mathrm{S}\left(x^{2}\right)=779 \cdot 1338, & \mathbb{S}(y z)=15421 \cdot 230 \\
\mathrm{~S}\left(y^{2}\right)=1336 \cdot 0155, & \mathbb{S}(z x)=10617 \cdot 960 \\
\mathrm{~S}\left(z^{2}\right)=190296 \cdot 0, & \mathbb{S}(x y)=751 \cdot 017 .
\end{array}
$$


Planes of Closest Fit to Systems of Points.

Using equation $(\beta)$ above, we quickly reach

$$
z=5 \cdot 4605 x+8 \cdot 4732 y \text {, }
$$

and therefore

$$
\mathrm{T}=1084-5 \cdot 4605 x-8 \cdot 4732 y . \quad . \quad .
$$

The values of $\mathrm{T}$ obtained by this formula are given in column 5 of Table II. The differences between these values and the experimental results are shewn in the next column. It will be seen from the figures that the fit is a good one except at the ends of the range. Had the last seven observations been omitted, $i$. e. had the amount of aluminium present in the alloy been less than $6 \%$, a linear law such as the above one would have agreed quite well with the observed results. As the authors of the original paper state that " the precise temperatures given in the table possess no very great significance," it seems quite reasonable to assume that the observations, up to $6 \%$ of aluminium, follow a linear law.

The sum of the squares of the deviations from the observed temperatures in this cuse is 1641.0210 , and the "root mean square" is 6.41 ". The sum of the squares of the deviations measured perpendicular to the plane can be obtained from the ahove figure by dividing by $\left\{(5 \cdot 186)^{2}+(8 \cdot 392)^{2}+1\right\}$, i. e. $102 \cdot 6120$. It is found to be $15 \cdot 9924$.

When the second method is used, the equation in $V_{m}$ is

$$
\begin{array}{lll}
779 \cdot 134-V_{m} & \multicolumn{1}{c}{751 \cdot 017} & 10617 \cdot 96 \\
751 \cdot 017 & 1336 \cdot 015-V_{m} & 15421 \cdot 23 \\
10617 \cdot 96 & 15421 \cdot 23 & 190296-V_{m}
\end{array} \mid=0 \text {. }
$$

This when expanded becomes

$$
\mathrm{V}_{m}^{3}-192411 \mathrm{~V}_{m}^{2}+52425892 \mathrm{~V}_{m}-786607941=0 \text {. }
$$

We want the least root of this cubic. It is quickly seen to be in the neighbourhood of 15 , and by successive approximations is found to be

very nearly.

$$
\mathrm{V}_{m}=15.9362,
$$

* The second decimal place was taken into account in finding this figure. This was done in order to compare with the results of $(\epsilon)$, which do not greatly differ from $(\gamma)$. 


$$
l x+m y+n z=0
$$

is the equation of required plane, the equations to find the ratios of $l, m$, and $n$ are

$$
\begin{aligned}
& 763 \cdot 198 l+751 \cdot 017 m+10617 \cdot 960 n=0, \\
& 751 \cdot 017 l+1320 \cdot 079 m+15421 \cdot 230 n=0 .
\end{aligned}
$$

From these we find

$$
\frac{l}{5+4908}=\frac{m}{85582}=\frac{n}{-1} .
$$

The equation of the plane is

and

$$
z=5 \cdot 4908 x+8 \cdot 5582 y
$$

$$
\mathrm{T}=1084-5 \cdot 4908 x-8 \cdot 5582 y . \quad \text {. . . }
$$

The temperatures given by this formula are shown in column 7 of the table, and the deviations from the observed values in column 8. They do not differ greatly from the results given by $(\gamma)$. The sum of the squares of the deviations in the table is 1658.9718 , which is, of course, greater than the corresponding number given by the first method. The "root mean square" is 6.44 , not greatly different from the first method value. Also $l^{2}+m^{2}+n^{2}$ becomes $104 \cdot 3923$. The actual sum of the squares of the deviations perpendicular to the plane is therefore 15.8917 , which is less than the value given by the first method, as it should be, but is not a very great improvement on it. Thus in this example, as in the last, the two metbods lead to very similar results.

III. For a third example we will take the case of a plane in three dimeusions to pass through two fixed points and to be closest fitting to a series of other points. The data for this case are taken from a railway time-table. The two fixed points are two terminal stations, and the variables are $x$, the distance (in miles) from one of these stations to some other station; $y$, the scheduled time (in minutes) allowed for a train between those stations; and $z$, the first-class single fare (in pence) between the stations. The figures are :-

$\begin{array}{rrr}r . & y . & z . \\ 30 & 49 & 69 \\ 52 & 80 & 117 \\ 60 & 97 & 135 \\ 69 & 115 & 156 \\ 81 & 136 & 182 \\ 100 & 164 & 224\end{array}$


the corresponding figures up to the other terminus being 114,187 , and 244 respectively. The figures should be expected to be fairly coplanar, and any formula obtained to represent them ought to give a good "fit." Four cases can be worked out here, viz. those obtained by making the sum of the square of the deviations in the directions of $x, y, z$ and perpendicular to the plane respectively a minimum. We find :

$$
\begin{array}{ll}
\mathrm{S}\left(x^{2}\right)=28526, & \mathrm{~S}(y z)=105264, \\
\mathrm{~S}\left(y^{2}\right)=76827, & \mathrm{~S}(z x)=64160, \\
\mathrm{~S}\left(z^{2}\right)=144311, & \mathrm{~S}(x y)=46801 .
\end{array}
$$

For the best fit in the direction of $z$, the equation of the plane will be

with the condition

$$
z=a x+b y
$$

$$
224=114 a+187 b . \cdot \cdot \cdot \cdot(\eta)
$$

In this case we have

$$
\Delta=\left|\begin{array}{ccc}
1 & 64160-114 \lambda & 105264-187 \lambda \\
64160-114 \lambda & 28526 & 46801 \\
105264-187 \lambda & 46801 & 76827
\end{array}\right| .
$$

Using the relations

$$
a=-\frac{\Delta_{10}}{\Delta_{00}}, \quad b=-\frac{\Delta_{90}}{\Delta_{00}},
$$

wa obtain

$$
\begin{aligned}
& a=2.2376+.00526 \lambda, \\
& b=.00706-.00077 \lambda .
\end{aligned}
$$

Substituting in $(\eta), \lambda$ becomes $-27 \cdot 2290$, and therefore

$$
\begin{aligned}
& a=2 \cdot 0943, \\
& b=.0281,
\end{aligned}
$$

and the best fitting plane in the direction of $z$ is

$$
z=2 \cdot 0943 x+\cdot 0281 y . . . \text {. . }
$$

In a similar manner we find the best fitting plane in the direction of $y$ is

$$
y=1 \cdot 6327 x+0036 z, . . . .
$$

and in the direction of $x$ it is

$$
y=0.0031 y+0.017 z . . \quad . \quad .
$$


When the deviations are measured in a direction perpendieular to the plane, the equation to determine $V_{m}$ (the least sum of the squares of these deviations) is, by $\$ 5$ above,

$$
\left|\begin{array}{cccc}
0 & 114 & 187 & 244 \\
114 & 28526-\mathrm{V}_{m} & 46801 & 64160 \\
187 & 46801 & 76827-\mathrm{V}_{m} & 105264 \\
244 & 64160 & 105264 & 144311-\mathrm{V}_{m}
\end{array}\right|=0
$$

the determinant being reversed for convenience in evaluation, i.e.

$$
107501 \mathrm{~V}_{n}^{2}-19413930 \mathrm{~V}_{n}+191936824=0,
$$

giving

$$
V_{m}=10 \cdot 49664
$$

Then

$d \propto\left|\begin{array}{cccc}1 & 114 \mu & 187 \mu & 244 \mu \\ 114 \mu & 28515 \cdot 5 & 46801 & 64160 \\ 187 \mu & 46801 & 76816.5 & 105264 \\ 244 \mu & 64160 & 105264 & 144301.5\end{array}\right|$

and

$$
l_{1}=-\frac{d_{10}}{d_{00}}, \quad l_{2}=-\frac{d_{20}}{d_{00}}, \quad l_{3}=-\frac{d_{03}}{d_{00}}
$$

where

$$
l_{1} x+l_{2} y+l_{3} z=0
$$

is the equation of the required plane. Since only the ratios of $l_{1}, l_{2}$, and $l_{3}$ are required, it is sufficient to find $d_{10}, d_{20}$, and $d_{30}$ (each of which contains $\mu$ as a factor). When we find these ratios we must divide each by $\left\{l_{1}^{2}+l_{2}^{2}+l_{3}^{2}\right\}^{\frac{2}{2}}$ in order to have the sum of their squares unity. In this way we find the equation of the plane is

$$
\cdot 8843 x-\cdot 4632 y-\cdot 0582 z=0 .
$$

The deviations of the results given by the formulæ $(\theta)$, $(\xi),(\xi)$, and $(\phi)$ from the actual values are (the deviation being positive when the formula gives a valuo greater than the actual value) :- 
Planes of Closest Fit to Systems of Points.

$(\theta)$

Deriation in direction of $z^{*}$.

$-4.795$

$-5 \cdot 850$

$-6 \cdot 619$

$-8.265$

$-8 \cdot 544$

$-9 \cdot 966$
(ל)

Deviation in direction of $y$.

$+\cdot 227$

$+5 \cdot 319$

$+1 \cdot 445$

$-1.787$

$-3 \cdot 101$

$+\cdot 070$ $(\xi)$

Deviation in direction of $x$.

$+\cdot 285$

$-2 \cdot 200$

$-178$

$+1.592$

$+2 \cdot 281$

$+.796$ $(\phi)$

Deviation perpendicular to plane.

$-\cdot 181$

$+2 \cdot 122$

$+\cdot 275$

$-1 \cdot 325$

$-1 \cdot 952$

$-\cdot 563$

The sum of the squares of these deviations are

(e) $341 \cdot 6570$,

(५) $43 \cdot 2458$,

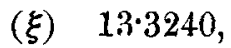

( $\phi$ ) 10.4942 (the exact value here should be.10*4966, the value of $V_{m}$ above).

The sum of the squares of the deviations given by $(\theta),(\zeta)$, and $(\xi)$ in directions perpendicular to those planes are found to be (by dividing the above values by the sum of the squares of the coefficients of the various equations) $63 \cdot 4239,11 \cdot 7969$, and 10.5767 respectively, all these, of course, being greater than the corresponding value given by $(\phi)$.

Equation $(\phi)$ can be written in the three forms :

$$
\begin{aligned}
& z=15 \cdot 2048 x-7 \cdot 9644 y \text {. . . . } \\
& y=1.9091 x-\cdot 1256 z \quad \cdot . \cdot . \\
& x=\cdot 5238 y+\cdot 0658 z \text {. . . . }
\end{aligned}
$$

These equations should be compared with $(\theta),(\zeta)$, and $(\xi)$ respectively. The sum of the squares of the deviations

* At first sight it seems remarkable that all the deviations given by $(\theta)$ are of the same sign, but a moment's consideration will show that this is quite possible. For a line in the plane is fixed, and the plane can only swing about this line. All the points may be on one side of the plane, but on either side of the line. Swinging the plane about the line to become closer (measured in a particular direction) to some of the points, therefore, may take it farther from some others. To verify $(\theta)$ the results given by the planes $z=2 \cdot 1404 x$ and $z=2 x+0856$, one on either side of $(\theta)$, were found. The sum of the squares of the deviations given by these formulæ were 341.6991 and $341 \cdot 8112$, both greater than the corresponding number for $(\theta)$. Thus $(\theta)$ gives a true minimum. 
given by these equations in the directions of $z, y$, and $x$ respectively are

$3102 \cdot 9568,48 \cdot 9069$, and $13 \cdot 4189$.

Comparing these with the results given by $(\theta),(\zeta)$, and $(\xi)$ above brings out clearly the fact that the plane which sati-fies one criterion for closest fit may give a very bad fit if measured by another criterion. This is particularly the case with $(\theta)$ and $\left(\theta^{\prime}\right)$, though $\left(\xi^{\prime}\right)$ is not greatly inferior to $(\xi)$ as the best fitting plane in the direction of $x$.

The Sir John Cass Technical Institute, London, E.C.

December 1910.

XLIV. A Method of Calitrating Fine Capillary Tubes. By Thomas Ralph Merton, B.Sc. (Oxon.)*

$7 \mathrm{HE}$ methods commonly used in the determination of the 1 bore of capillary tubes are direct optical measurement of the bore at the orifice, or weighing a drop of mercury which occupies a known length in the capillary. When very fine capillaries, having an internal diameter of the order of $-1 \mathrm{~mm}$., are to be measured, these methods present serious difficulties.

For many purposes it is necessary to obtain a value of the mean bore, and as no glass capillary is uniform for any ccnsiderable length, a measurement of the hore at the orifices is liable to serions error. The weight of a column of mercury $10 \mathrm{~cm}$. long contained in a capillary tube of $\cdot 1 \mathrm{~mm}$. bore is about $6.01 \mathrm{grm}$., so that to obtain an accuracy of 1 per cent. the weighing must be correct to $0.1 \mathrm{mgrm}$.

The following experiments were performed with the object of investigating the accuracy with which a measurement of the electrical resistance of a fine glass capillary filled with mercury can be made. From this a mean value of $r^{2}$ (where $\boldsymbol{r}$ is the internal ridius) can be calculated.

The first series of experiments was conducted in a large water-bath, containing about 30 litres, kept at $18^{\circ} \mathrm{C}$. by an electric-filament lamp which was governed by a large spiral toluene regulator; and other experiments were performed in a bath kept at $25^{\circ}$ by a small gas-flame governed by a fluted toluene regulator. In both baths the temperature could be kept constant to $0^{\circ} .01 \mathrm{C}$.

* Communicated by the Author. 